\title{
On Usage of Adaptive Filter in Acoustic Echo Cancellation for Voice Communication
}

\author{
Ning Cai ${ }^{a}$, ChunLin Deng *b \\ College of Electrical Engineering, Northwest University for Nationalities, Lanzhou, China \\ accaining91@tsinghua.org.cn_ bdengchunlin_ray@163.com
}

\section{Keywords: Acoustic Echo; Adaptive Filter; RLS; LMS}

\begin{abstract}
Acoustic echo is a common phenomenon in voice communication. The interference signal caused by acoustic echo will greatly reduce the quality of voice transmission. Thus, techniques should be applied to diminish acoustic echo. This paper endeavors to introduce the application of adaptive filter in acoustic echo cancellation. Adaptive filter can dynamically adapt its parameters to minimize the difference between its output and the actual echo signal so that the actual echo signal can be eliminated as much as possible. Furthermore, this paper elaborates the operational process of certain common filtering algorithms of adaptive filter and performs comparison of the performance of these filtering algorithms.
\end{abstract}

\section{Introduction}

The echo will bring the result that the voice signal recipient cannot exactly receive the information in the voice signal transmission process so that the quality of communication will be greatly reduced. Therefore, the echo cancellation is very important in the voice transmission process which needs high quality, for example, phone with hands-free function, digital court, conference in large venue, conference call, etc. It can be forecasted that the echo cancellation has a very broad market prospect in the future. The adaptive filter, as the core of echo cancellation, can acquire the predicted echo signal through simulating the actual echo path so that the actual echo signal can be eliminated as much as possible [1-3]. This paper mainly addresses on introducing the application of adaptive filter in echo cancellation system and elaborating some frequently-used filtering algorithms of linear adaptive filter. Overall, the significance of this paper is to lay a solid theoretical foundation for the application of the adaptive filter in an actual echo cancellation system.

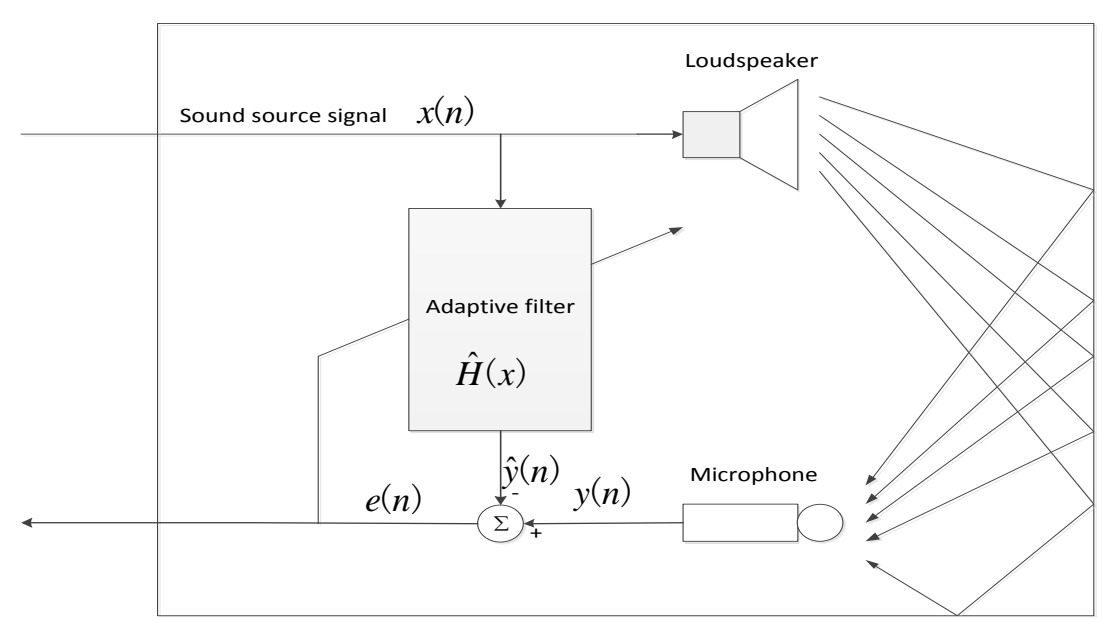

Fig. 1 Operational principle of adaptive filter in an acoustic echo cancellation system 


\section{Application of Adaptive Filter in Acoustic Echo Cancellation}

At present, the adaptive filter technology is a relatively mature technology in echo cancellation. The input of adaptive filter is same with the loudspeaker. The output of loudspeaker will be collected again by microphone after being reflected many times. The function of adaptive filter is to simulate the actual echo path exactly and to acquire the predicted echo signal so that the final input signal of loudspeaker which is the difference of the predicted echo signal and the actual echo signal can gradually become zero [4]. The operational principle of adaptive filter in an acoustic echo cancellation system is illustrated in Fig. 1.

In Fig. 1, the echo path $H(x)$ can be treated as a function, and it depends on the actual environment. The adaptive filter can also be treated as a function ${ }^{\hat{H}(x)}$, and it should approach $H(x)$ as close as possible. They should produce the same result when their input is identical. The adaptive filer can adjust its parameters with the actual environment, which means that $\hat{H}(x)$ can keep on simulating $H(x)$. The output of filter is $\hat{y}(n)$. The ideal error $e(n)=d(n)-\hat{y}(n)$ should be zero, with ${ }^{e(n)}$ as the actual output, which implies that the echo has been eliminated thoroughly.

\section{Adaptive Filter}

The filter can be divided into two types according to the relationship between the output and the input of filter: linear filter and nonlinear filter. Adaptive filter, as an important branch of filter, can vary its filtering parameters in correspondence with the actual acoustic echo path. The updating method of these filtering parameters is different when the adaptive filter adopts different filtering algorithm.

The recursive algorithm, as a frequently-used algorithm of adaptive filter, can still complete filtering operation exactly when the information which derives from input signal is incomplete. The operational step of recursive algorithm is that it acquires a sort of optimal solutions after a series of successful iterating operations from some kind of assigned initial condition sets when these initial conditions are steady. Furthermore, it can still track the input signal which alters with time slowly and acquire a sort of optimal solutions after a series of successful iterating operations when these initial conditions are unsteady [5].

These parameters of recursive algorithm are related to the actual acoustic echo path when the adaptive filter is operating. Thus, they will be updated from one iteration to another until the adaptive filter can simulate the actual acoustic echo path.

\section{Linear Adaptive Filter}

The linear adaptive filter, as a branch of adaptive filter, adopts the linear filtering algorithm to complete the filtering operation. The linear filtering algorithm includes two fundamental operating processes: filtering process and adapting process. The objective of filtering process is to obtain the output signal. Adapting operation is to vary these parameters of filtering algorithm.

The linear adaptive filter possesses memory, which depends on its impulse response. The linear filter can be divided into two types according to the impulse response: finite impulse response filter (FIR) and infinite impulse response filter (IIR). The structure of FIR filter only has the feedforward path. However, the structure of IIR filter also has the feedback path. FIR filter and IIR filter can be characterized respectively by the finite memory and infinite but attenuated memory.

Although the computational complexity of IIR filter is lower than FIR filter. But the FIR filter is still applied in most actual applications for its better stability.

The transversal filter, as a type of FIR filter, consists of a series of multipliers, delay units and adders. Its structure is shown in Fig. 2. The number of unit delays determines the duration of the impulse response and the order of transversal filter. The order of transversal filter is denoted by $N$ in Fig. 2. The unit delay operator is expressed by $Z^{-1}$ in Fig. 2 . The output signal is ${ }^{x(n-1)}$, which is the result of input signal operated by $Z^{-1}$ 
operation. The primary role of multiplier is to multiply these parameters of filter with the corresponding input signal. The parameter of filter is also called tap weights $w_{i}(i=0,1,2, \ldots, N-1)$. The output of multiplier connected to the input signal ${ }^{x(n-i)}$ is $w_{i}(n) x(n-i)$. The adder of filter is employed to combine the output signal of each multiplier so that the finial output signal of filter can be produced.

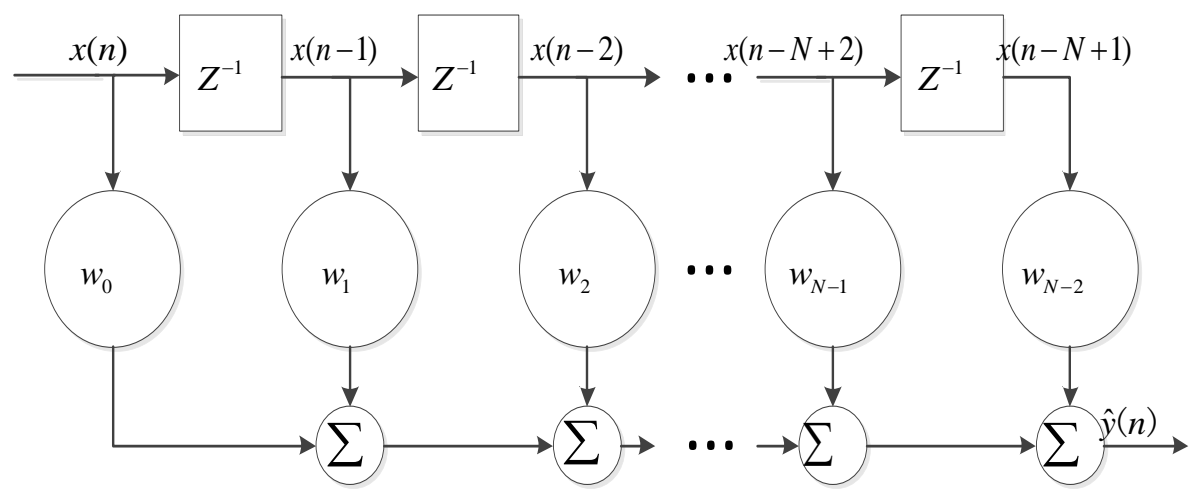

Fig. 2 Structure of Transversal Filter

The output of the transversal filter shown in Fig. 2 is:

$$
\hat{y}(n)=\sum_{i=0}^{N-1} w_{i}(n) x(n-i)
$$

$n$ is the time the predicted signal appears; $N$ is the order of transversal filter; and $w_{0}, w_{1}, \ldots, w_{N-1}$ are $N$ adjustable parameters.

The concrete structure of transversal filter depends on the value of $N$ adjustable parameters $w_{0}, w_{1}, \ldots, w_{N-1}$. The adaptive filter can change the value of these parameters online, which are usually denoted by a filter parameter vector:

$$
W(n)=\left[w_{0}(n) w_{1}(n) \ldots w_{N-1}(n)\right]^{T}
$$

The value of these parameters depends on the difference between the actual echo signal and predicted echo signal. The filter can well simulate the actual echo path when the actual echo signal is equal to the predicted echo signal, and it is not necessary to update the value of these parameters $w_{0}, w_{1}, \ldots, w_{N-1}$ anymore. Perhaps, the filter will need an adapting process to adjust the parameter vector until it become appropriate when the echo cancellation system starts to run or the echo path changes with the actual environment [6-8].

\section{Algorithm of Linear Adaptive Filter}

The derivation method of recursive algorithm applied in linear adaptive filter can be divided into two types: recursive least squares estimation and stochastic gradient algorithm.

Recursive Least Squares Estimation. The recursive least squares estimation (RLS) is based on the least squares method. The least squares can be represented by recursive estimation and block estimation. The data flow in block estimation is arranged with blocks, and the length of these data blocks is identical. The updating method of recursive estimation is to sample data individually. Generally, the storage capacity needed for block estimation is more than recursive estimation. Therefore, the recursive estimation is widely used in reality.

The recursive least squares estimation can be divided into three categories:

1) The standard RLS algorithm: The standard RLS algorithm is derived according to the matrix inverse theorem in linear algebra under the premise that the basic structure of linear adaptive filter is transversal filter. The main defect of this algorithm is that its computational process is very complex and not numerically robust. 
2) The squared RLS algorithm: The squared RLS algorithm applies the QR decomposition method to decompose the input signal matrix into a regular orthogonal matrix and an upper triangular matrix before processing these data.

3) The fast RLS algorithm: The primary feature of the fast RLS algorithm is that the operating speed is faster than another RLS algorithm.

The RLS algorithm can still be convergent quickly when the divergence extent of eigenvalue of input signal matrix is larger. But this operation will greatly increase the computational complexity. Thus, the RLS algorithm is rarely employed in reality.

Stochastic Gradient Algorithm. The stochastic gradient algorithm is realized under the premise that the basic structure of adaptive filter is transversal. The quadratic function of tap weights of filter is the cost function of transversal filter. The dimensional paraboloid of minimum point is the only method to determine the relationship between the mean square error and unknown tap weights of filter, and this paraboloid is called error performance curve. The tap weights corresponding with minimum point of error performance curve is defined as the optimal solution. The cost function is also called mean square error when the input signal is relatively steady.

The least mean square algorithm (LMS) is a kind of relatively mature and widely-used stochastic gradient algorithm. LMS algorithm can quickly and easily acquire a satisfied result when these assigned condition sets are correct. But the nonignorable defect of LMS algorithm is that its convergence speed is slow and the ratio between the largest eigenvalue and smallest eigenvalue is sensitive to the input tap. Despite this, the LMS algorithm is still used widely for its advantages.

It is a major feature of error performance curve that its direction will change with time, and LMS algorithm has an additional mission that it must track the minimum point of error performance curve continuously. Actually, if the change speed of input signal is lower than the study speed of LMS algorithm, the LMS algorithm can exactly track the input signal [9-10].

Overall, the LMS algorithm is easy to realize, and its storage required and calculating complexity in each iteration is very small. Therefore, it is as relatively ideal filtering algorithm of adaptive filter.

\section{Conclusions}

With the extensive application of voice signal transmission, one of the major problems in voice signal transmission is the echo. The adaptive filter can build a model to simulate the actual acoustic echo path so as to acquire the output which is almost similar with the echo collected again by microphone such that the actual acoustic echo is eliminated as much as possible. The different filtering algorithm introduced in this paper has its own pros and cons. But considering all aspects of the performance that adaptive filter requires, the LMS algorithm is a relatively ideal filtering algorithm in an actual acoustic echo cancellation system.

\section{Acknowledgements}

This work is supported by Program for Young Talents of State Ethnic Affairs Commission (SEAC) of China (Grant [2013] 231), and by National Natural Science Foundation (NNSF) of China (Grants 61263002 \& 61374054).

\section{References}

[1] J. Homer, Adaptive Echo Cancellation in Telecommunications, Doctoral Dissertation, Australian National University (University of Newcastle), 1994. 
[2] L. S. H. Ngia, System Modeling Using Basis Functions and Application to Echo Cancellation, Doctoral Dissertation, Chalmers University of Technology, 2000.

[3] S. Furui and M. M. Sondhi, Advances in Speech Signal Processing, Marcel Dekker Inc., 1992.

[4] J. Benesty, T. Gänsler, D. R. Morgan, M. M. Sondhi, S. L. Gay, Advances in Network and Acoustic Echo Cancellation, Springer Verlag, 2001.

[5] M. L. Honig, D. G. Messerschmitt, Adaptive Filters: Structures, Algorithms and Applications, Springer Verlag, 1984.

[6] S. Haykin, Adaptive Filter Theory, Prentice Hall, 1996.

[7] M. Bellanger, G. Maurice, Adaptive Digital Filters, Marcel Dekker Inc., 2001.

[8] C.F.N. Cowan, P.M. Grant, P.F. Adams, Adaptive Filters, Prentice Hall, 1985.

[9] P. S. R. Diniz, Adaptive Filtering, Springer Verlag, 1997.

[10] B. Farhang-Boroujeny, Adaptive Filters: Theory and Applications, John Wiley \& Sons, 2013. 